\title{
What about superfertility, decidualization, and natural selection?
}

\author{
Carolyn Coulam ${ }^{1}$
}

Received: 9 December 2015 / Accepted: 7 January 2016 / Published online: 3 February 2016

(C) Springer Science+Business Media New York 2016

Recurrent pregnancy loss, defined as two or more failed pregnancies by the American Society for Reproductive Medicine [1], is a clinical problem because known effective treatment is limited. Effective treatment depends on the cause of the reproductive failure. Thus, attention has been focused on determining causes of recurrent pregnancy loss. Even so, to date, only chromosomal abnormalities of the conceptus and immunologic risk factors have been generally accepted as etiologies of recurrent pregnancy loss. Recently, a novel pathologic pathway that involves impaired decidualization of endometrial stromal cells has been proposed as a cause of recurrent pregnancy loss [2]. Further, it has been hypothesized that the impaired decidualization is manifested by the prolongation of the window of implantation allowing for increased fecundity and "superfertility" [2-7]. The following paragraphs will describe the evidence for these notions.

\section{Decidualization}

Decidualization of the endometrium is essential for successful implantation of all species in which the blastocyst breaches the uterine epithelium [8]. It is characterized by secretory transformation of the uterine glands, influx of specialized

Capsule It is becoming increasingly apparent that the causes of recurrent pregnancy failure go well beyond previously held notions of embryo chromosome abnormalities when viewed in the context of natural selection rather than the consequences of human ART treatments.

Carolyn Coulam

cbcoulam@aol.com

1 Reproductive Medicine Institute, 2500 Ridge Ave, Suite 200, Evanston, IL 60201, USA uterine natural killer cells, vascular remodeling, and morphologic and biochemical reprogramming of the endometrial stromal cells. Major secretory products of decidual stromal cells include prolactin and insulin-like growth factor binding protein-1 (IGFBP-1), two proteins that have been used as markers of decidualization [9]. In humans, decidualization begins approximately 6 days after ovulation at the onset of the putative window of implantation which is thought to last not more than 2-4 days [10]. One effect of abnormal decidualization has been postulated to result in a prolongation of the window of implantation [2-7]. Biochemical support for this concept is provided by studies of cultured endometrial stromal cells from women experiencing recurrent miscarriage both in vitro and in vivo [3]. Analyses of midsecretory endometrial biopsies from women with and without a history of recurrent pregnancy loss demonstrated that recurrent pregnancy loss is associated with decreased expression of the decidual marker prolactin and an increased expression of prokinectin-1, a cytokine that promotes implantation [3]. These in vivo findings were confirmed in vitro when endometrial stromal cells from women with and without recurrent miscarriages were decidualized in culture [3]. These co-culture experiments provided evidence that impaired decidualization prolonged the window of implantation [4]. The consequences of prolonged window of receptivity would be expected to (1) facilitate delayed implantation of compromised embryos and (2) increase fertility or the probability of achieving pregnancy within one menstrual cycle.

\section{Fertility}

Fertility rates can be measured as the time taken to achieve pregnancy expressed as monthly fecundity rates (MFRs), that is, the probability of achieving pregnancy within one 
menstrual cycle [11]. While age dependent, the average MFR in humans has been reported as $20 \%$, moderate and severe infertility as MFR of 5 and $1 \%$, respectively, and superfertility as MFR of $60 \%$ or greater [11]. Using a mathematical model, it has been estimated that $79 \%$ of the population is fertile, $18 \%$ subfertile, and $3 \%$ superfertile $[11,12]$. Using the model of Tietze [12], two studies have shown that $32-40 \%$ of women experiencing recurrent pregnancy loss are superfertile [3, 13]. When the 32-40\% prevalence of superfertility among women with a history of recurrent pregnancy loss was compared with the $3 \%$ prevalence in the general population [11, 12], the difference was significant $(P<0.0001)$ [13]. Confirmation of the association of superfertility and recurrent pregnancy loss adds clinical support to the concept that impaired decidualization resulting in prolongation of the window of implantation is a cause for recurrent miscarriages. Prolongation of the window of implantation could lead to implantation of developmentally delayed or compromised embryos.

\section{Karyotype of products of conception}

The most common abnormality found in developmentally compromised preimplantation embryos as well as aborted products of conception is an abnormal chromosome complement [14-16]. A recent study of products of conception from 200 women experiencing recurrent miscarriages revealed a $20 \%$ chromosomal abnormality rate [13]. When this study was expanded to look at results of 309 karyotypes from 420 women with a history of recurrent miscarriage, $25 \%$ (78/309) displayed abnormal chromosome complement and $75 \%$ (231/ 309) were normal [14]. Among the 231 normal karyotypes, $53 \%(121 / 231)$ were $46, X X$ and $47 \%(109 / 231)$ were $46, X Y$ suggesting that the high rate of chromosomal normality was not in large part explained by maternal contamination [13, 14]. Results of chromosome analysis from abortus material from women experiencing recurrent miscarriages reported in the literature are shown in Table 1. Aneuploidy rates have varied from 20 to $78 \%$ [13-23]. All reports of aneuploidy rates greater than $30 \%$ were obtained from a population of abortuses sent to the chromosome analysis laboratories [15, $16,18]$ in contrast to those obtained from a population of recurrent aborting women [13, 14, 17, 19]. If the prevalence of abnormal concepti among women with a history of recurrent pregnancy loss is the question, then the population women of recurrently aborting must be studied rather than the products of conception. Investigating the products of conception for obstetrical history of the women who aborted will provide information regarding the sensitivity and specificity. One of the highest chromosomal abnormality rates observed when comparative genomic hybridization (CGH) was used for the analysis rather than cytogenetics [23]. The explanation for
Table 1 Results of chromosome analysis from abortus material from women experiencing recurrent miscarriage

\begin{tabular}{llll}
\hline Study & Number & $\begin{array}{l}\text { Mean age } \\
\text { (year) }\end{array}$ & $\begin{array}{l}\text { \% Abnormal } \\
\text { karyotype POC }\end{array}$ \\
\hline Stern [15] & 224 & 35 & 57 \\
Ogasawara [16] & 234 & 31 & 51 \\
Carp [17] & 125 & 32 & 29 \\
Stephenson [18] & 420 & 34 & 46 \\
Sullivan [19] & 255 & 31 & 25 \\
Marquard [20] & 137 & 39 & 78 \\
Grande [21] & 376 & 35 & 60 \\
Sugiura-Ogasawara & 482 & 32 & 41 \\
[22] & & & 66 \\
Robberect [23] CGH & 51 & - & 20 \\
Orlando [13] & 192 & 35 & 25 \\
Coulam [14] & 420 & 35 & \\
\hline
\end{tabular}

increased abnormality rate given was the fact that $\mathrm{CGH}$ can detect microdeletions and microduplications across the genome [23]. The problem with this explanation is that the frequencies of these microdeletions among embryos resulting in live birth are not known. Recurrent aneuploidy occurred in $10 \%$ (19) and $14 \%$ (data not shown) of patients experiencing recurrent pregnancy loss who had two or more miscarriages karyotyped. When comparing the frequencies of all karyotypes of products of conception from women with a history of recurrent pregnancy loss with those reported from all spontaneous abortions [24, 25], a significant difference $(P<0.0001)$ is observed.

Thus, the contribution of abnormal concepti as a cause of recurrent pregnancy loss may have to be reassessed. While an abnormal chromosome complement of the conceptus has been accepted as the most common cause for all miscarriages, uterine causes have accounted for almost half of all miscarriages [26].

\section{Conclusion}

Recurrent pregnancy loss is associated with impaired decidualization leading to a significantly higher prevalence of superfertility than the normal population. Abortuses from women with a history of recurrent pregnancy loss display a higher prevalence of chromosomally normal pregnancy losses compared with sporadic abortions. It had been proposed that in view of the high incidence of gross chromosomal errors in human preimplantation embryos, decidualization is a means of natural selection of embryos limiting maternal investment of impaired pregnancies [2-7]. If our data can be confirmed, natural selection of embryos for implantation would have to 
include embryos developmentally compromised for reasons other than chromosomal abnormalities.

By restricting the window of implantation, the continuously changing endometrial environment is aligned to meet the requirements of an implanting blastocyst. Prolonged endometrial receptivity carries a risk of implantation of developmentally delayed embryos thus facilitating non-synchronized embryo implantation in an unsupportive environment. In addition, asynchrony between endometrial and embryo development in early pregnancy may trigger a spectrum of pathological events, leading to miscarriage or predispose for obstetrical complications associated with defective placentation, such as preeclampsia, fetal growth restriction, and preterm birth [27].

Decidualization has also been suggested as a means of providing natural selection of embryos to limit maternal investment of impaired pregnancies [2-7]. Co-culture experiments have shown that decidual cells sense signals from developmentally compromised embryos and respond by shutting down the secretion of cytokines necessary for implantation [2]. Delayed implantation could negate embryo quality control and cause early placental failure, regardless of the embryonic karyotype. This pathological pathway provides an explanation for the observation that some patients experiencing recurrent pregnancy loss seem exceptionally fertile, often conceiving within one or two cycles. Thus, as the clinical correlate of inappropriate uterine receptivity, "superfertility" should be considered as a genuine reproductive disorder that requires targeted intervention. However, little information is available regarding the molecules that signal closure of the window of implantation [28]. The expression of a gene of the TGF family called endometrial bleeding associated factor (ebaf) has been identified only in late secretory and not in the proliferative, early, or midsecretory phases of the menstrual cycle [29]. In situ hybridization revealed the expression was primarily confined to mesenchymal cells of the endometrial stroma rather than the epithelium or endothelium [29]. Ebaf was shown to be prematurely increased during the window of implantation in a subset of infertile patients and its overexpression to inhibit the expression of IGFBP-1 and prolactin, key decidual proteins [30]. Whether under expression of ebaf results in prolongation of the window of implantation requires further study.

\section{References}

1. Practice Committee of the American Society for Reproductive Medicine. Definitions of infertility and recurrent pregnancy loss. Fertil Steril. 2008;90:60S.

2. Teklenburg G, Salker M, Molokhia M, Lavery S, Trew G, Aojanepong T, et al. Natural selection of human embryos: decidualizingendometrial stromal cells serve as sensors of embryo quality upon implantation. PLoS One. 2010;5:e1028.
3. Salker M, Teklenburg G, Molohkia M, Lavery S, Trew G, Aojanepong T, et al. Natural selection of human embryos: Impaired decidualization of endometrium disables embryomaternal interactions and causes recurrent pregnancy loss. PLoS One. 2010;5:e10287.

4. Salker MS. Disordered IJ33/ST2 activation in decidualizing stromal cells prolongs uterine receptivity in women with recurrent pregnancy loss. PLoS One. 2012;7:e52252.

5. Teklenburg G, Salker M, Heijnen C, Macklon N, Brosens J. The molecular basis of recurrent pregnancy loss: impaired natural embryo selection. Mol Hum Reprod. 2010;16: e10258.

6. Quenby S, Vince G, Farquharson R, Alpin J. Recurrent miscarriage: A defect in nature's quality control? Hum Reprod. 2002;17:1959 63 .

7. Weimer CHE, Kavelaars A, Brosens J, Gellersen B, de VreedenElbertse T, Heijnen CJ, et al. Endometrial stromal cells of women with recurrent miscarriage fail to discriminate between high and low quality human embryos. PLoS One. 2010;7:e4144.

8. Brosens JJ, Pijnenborg R, Brosens IA. The myometrialjunctional zone spiral arteries in normal and abnormalpregnancies: a review of the literature. Am J Obstet Gynecol. 2002;187:1416-23.

9. Gellersen B, Brosens J. Cyclic AMP and progesterone receptor cross-talk in human endometrium: a decidualizing affair. J Endocrinol. 2003;178:357-72.

10. Wilcox AJ, Baird DD, Weinberg CR. The time of implantation of the conceptus and loss of pregnancy. N Engl J Med. 1999;340: 1796-9.

11. Evers JL. Female subfertility. Lancet. 2002;360:151-9.

12. Tietze C, Guttmacher AF, Rubin S. Time required for conception in 1727 planned pregnancies. Fertil Steril. 1950;1:338-46.

13. Orlando J, Coulam C. Is superfertility associated with recurrent pregnancy loss? Am J Reprod Immunol. 2014;72:549-54.

14. Coulam C. Association between immunologic causes and superfertility among women experiencing recurrent pregnancy loss. Am J Reprod Immunol. 2015;73:1.

15. Stern J, Coulam CB. Frequency of abnormal karyotypes among abortuses from women with and without a history of recurrent spontaneous abortion. Fertil Steril. 1996;65:250-3.

16. Ogasawara M, Aoki K, Okada S, Suzumori K. Embryonic karyotype of abortuses in relation to the number of previous miscarriages. Fertil Steril. 2000;73:300-4.

17. Carp H, Toder V, Aviram A, Daniely M, Mashiach S, Barkai G. Karyotype of the abortus in recurrent miscarriage. Fertil Steril. 2001;75:678-82.

18. Stephenson MD, Awartani KA, Robinson WP. Cytogenetic analysis of miscarriages from couples with recurrent miscarriage: a casecontrol study. Hum Reprod. 2002;17:446-51.

19. Sullivan A, Silver RM, LaCoursiere DY, Porter TF, Branch DW. Recurrent fetal aneuploidy and recurrent miscarriage. Obstet Gynecol. 2004;104:784-8.

20. Marquard K, Westphal LM, Milki AA, Lathi RB. Etiology of recurrent pregnancy loss in women overthe age of 35 years. Fertil Steril 2010; 94:1473-1477.

21. Grande M. Borrell A, Garcia-Posada R, Borobio V, Munoz M, Creus M, Soler A, Sanchez A, Balasc J. The effect of maternal age on chromosomal anomaly rate and spectrum in recurrent miscarriage. Hum Reprod 2012;27:3109-3117.

22. Sugiura-Osagawara, Ozaki Y, Katano K, Suzumori N, Kitaori T, Mizutani E. Abnormal embryonic karyotype is the most frequent cause of recurrent miscarriage. Hum Reprod 2012;27:2297-2303

23. Robberect C, Pexsters A, Fryns JP, D'Hooghe T, Vermeesch JR. Cytogenetic and morphologic analysis of early productsof conception following hysteron-embryoscopy from couples with recurrent pregnancy loss. Prenat Diagn. 2012;32:933-42. 
24. Boue A, Boue J, Gropp A. Cytogenetics of pregnancy wastage. Adv Hum Genet. 1985;14:1-57.

25. Hassold T, Chen N, Funkhouser J, Jooss T, Manuel B, Matsuura J, et al. A cytogenetic study of 1000 spontaneous abortions. Ann Hum Genet. 1980;44:151-64.

26. Clark DA, Daya S, Coulam CB, Gunby J. Recurrent Miscarriage Immunotherapy Trialists Group: implication of abnormal humantrophoblast karyotype for the evidencebased approach to the understanding, investigation, and treatment of recurrent spontaneous abortion. Am J Reprod Immunol. 1996;35:495-8.
27. Kaufman P, Black S, Hupperts B. Endovascular trophoblast invasion: implications for the pathogenesis of intrauterine growth retardation and preeclampsia. Biol Reprod. 2001;69:1-7.

28. Tabibzadeh S. Molecular control of the implantation window. Hum Reprod Update. 1998;5:465-51.

29. Kothapalli R, Buyuksal I, Wu SQ, Chegini N, Tabiazadeh S. Detection of ebaf, a novel human gene of the transforming growth factor beta family association of gene expression with endometrial bleeding. J Clin Invest. 1997;99:2342-50.

30. Tabibzadeh S. Isolation, characterization and function of EBAF/ LEFTY B: role in infertility. Ann NY Acad Sci. 2011;1221:98-102. 